\title{
An Investigation of a Method for the Analysis of Smokes According to Particle Size ${ }^{1}$
}

\author{
William N. Lipscomb, ${ }^{2}$ T. R. Rubin, ${ }^{3}$ and J. H. Sturdivant \\ California Institute of Technology, Pasadena, California ${ }^{4}$
}

(Received August 2, 1946)

\begin{abstract}
An electrical precipitation method for the analysis of smokes according to particle size has been investigated. A thin stream of smoke particles moving under laminar conditions in a wind tunnel of rectangular cross section is electrically charged in a small region. The charged particles are then precipitated by the uniform field existing between the charged upper plate and the grounded lower plate of the wind tunnel as a long track along the lower plate. The sizefrequency distributions of the particles precipitated at various points on the lower plate were determined from measurements on enlargements of electron-microscope photographs of samples of the smoke precipitated at various points. The agreement between theory and experiment is fair, but the range of sizes of the particles precipitated at a given point is rather large.
\end{abstract}

\section{INTRODUCTION}

$T$ HE use of a precipitation method for the estimation of sizes of smoke particles was first described by Rohmann. ${ }^{5}$ His apparatus consisted of a wind tunnel of rectangular cross section with an electric field between the negatively charged upper plate and the grounded lower plate. The smoke was introduced in to the wind tunnel through a small tube and was charged in a small region by a corona discharge from a point. The charged particles were precipitated by the uniform field as a track about $5 \mathrm{~cm}$ in length. The size of the particles was measured with an optical microscope and compared with the particle radius calculated from the theory of precipitation of charged spherical particles in a uniform field. Rohmann did not report the sizefrequency distributions of the particles precipitated at various points, but only the average size in the densest region of the precipitate. No statement was made regarding the regularity of shape of the metallic oxide particles in the smokes which he used for his investigation. Rohmann mentions two difficulties encountered in his experiments. The first was the difficulty of obtaining laminar gas flow in the wind tunnel;

1 This work was done in whole under the Contract No. OEMsr-103, Supplement 1, between the California Institute of Technology and the Office of Scientific Research and Development, which assumes no responsibility for the accuracy of the statements contained herein.

2 Present address: School of Chemistry, University of Minnesota, Minneapolis 14, Minnesota.

${ }^{3}$ Present address: Department of Chemistry, Ohio State University, Columbus, Ohio.

${ }^{4}$ Contribution No. 1054.

5 H. Rohmann, Zeits. f. Physik 17, 253 (1923). even in the absence of a charging corona he was forced to work with gas velocities of nearly 40 $\mathrm{cm} / \mathrm{sec}$. in order to obtain a stable thread of smoke. The second difficulty was the disturbance of the thread of smoke by the electrical wind which accompanies corona discharge; he was therefore constrained to use comparatively small charging currents.

The present investigation consists of a more extensive study of the precipitation method for the analysis of smokes according to particle size. A modified form of the apparatus described by Rohmann was used. The rectangular wind tunnel was redesigned in order to give essentially laminar flow at gas velocities of approximately $10 \mathrm{~cm} / \mathrm{sec}$. Since the point corona was found to be unsatisfactory, other methods of obtaining charging currents were investigated. The electron microscope was used to record the sizes of particles precipitated at various points in the apparatus. Had the separation of the smoke particles proved more satisfactory a much more rapid method was to be used (based on calibration with the electron microscope results) for the determination of the size-frequency distribution function; this proposed method consisted of measurement of the current carried by the charged smoke particles to small metal collectors mounted in the plane of the lower plate and insulated from it. Because the results were not completely satisfactory, however, only the studies of various charging devices, representative size-frequency distribution curves, and com- 


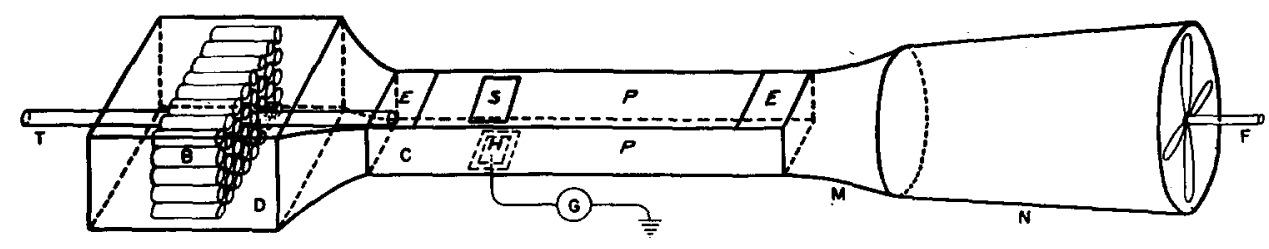

Perspective

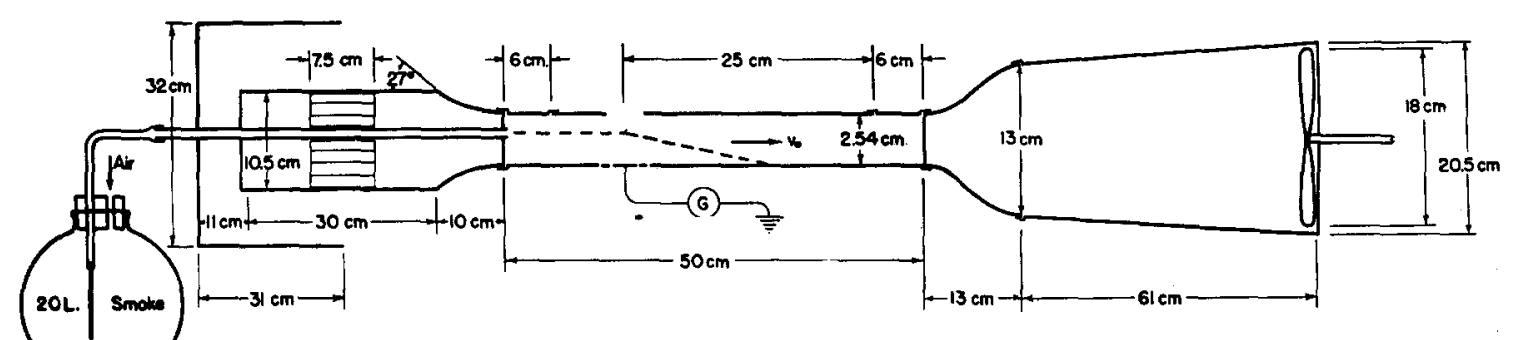

Elavation

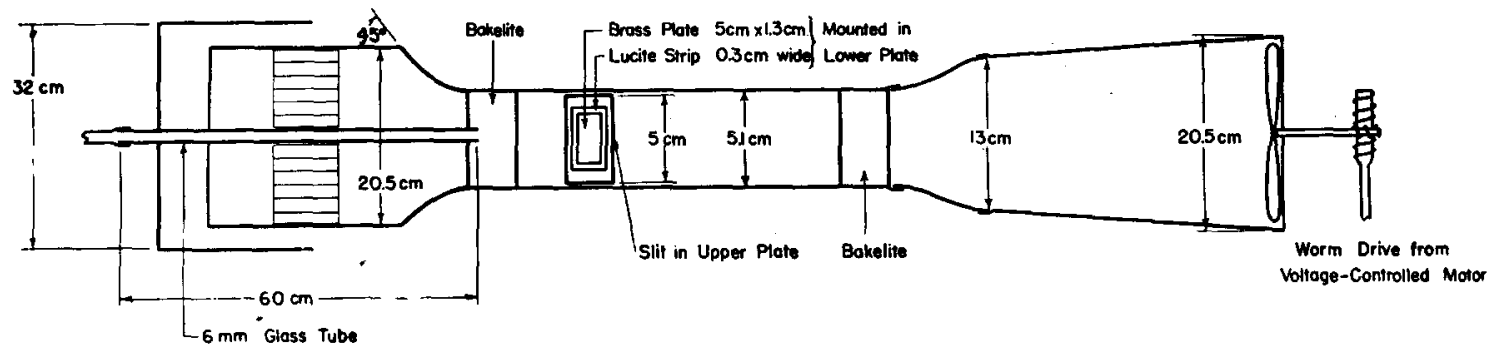

FIG. 1. The precipitation apparatus.

parisons of experimental results with theoretical precipitation curves are presented here.

\section{APPARATUS}

A diagram of the precipitation apparatus is shown in Fig. 1. The smoke, which was contained in a large flask, was forced by means of compressed air first through a short length of smallbore glass tubing (to reduce turbulence) and then in to the entrance tube $T$. This tube extended through the entrance duct $D$ to the rectangular precipitation chamber $C$. In order to minimize turbulence the entrance duct was provided with a honeycomb baffle $B$ made from short lengths of Cellophane soda straws. Further reduction of turbulence was obtained by placing a box in front of the entrance duct in the position indicated in the plan and elevation of Fig. 1. The 


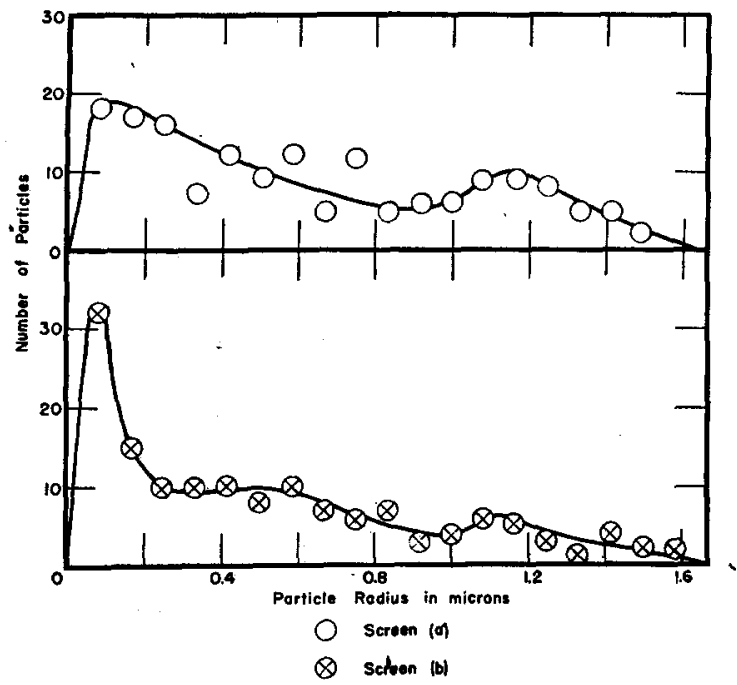

FIG. 2a. Size-frequency distributions of particles in a rosin smoke charged by means of a heated filament.

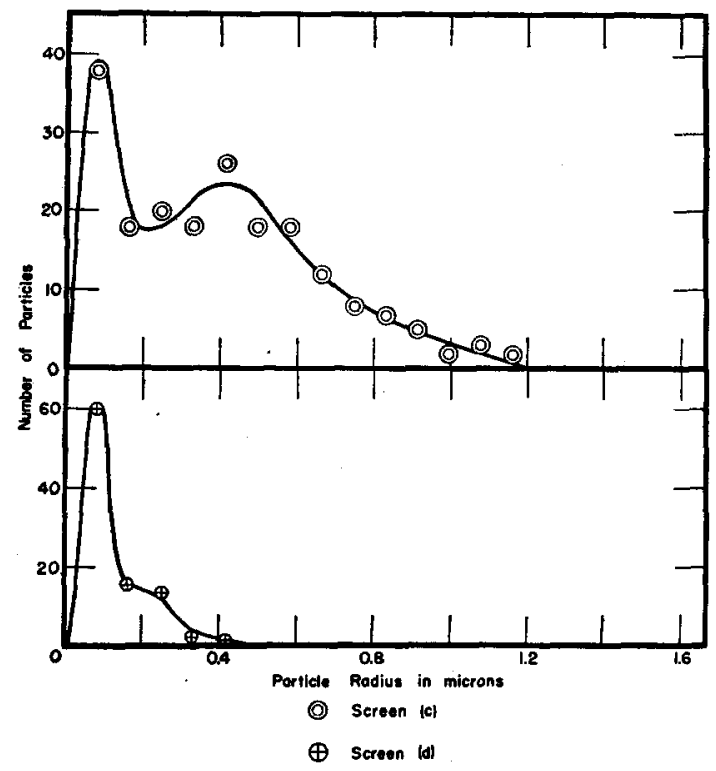

FIG. 2b. Size-frequency distributions of particles in a rosin smoke charged by means of a heated filament.

precipitation chamber consisted of two parallel brass plates $P$ separated by lucite strips. The upper plate, which was charged to about $-4 \mathrm{kv}$, was insulated from the entrance and exit ducts by bakelite strips $E$. The various charging devices which were tested were placed above or in the plane of an aperture $S$ in the upper plate, and were surrounded by an air-tight insulator to prevent air from entering around the charging

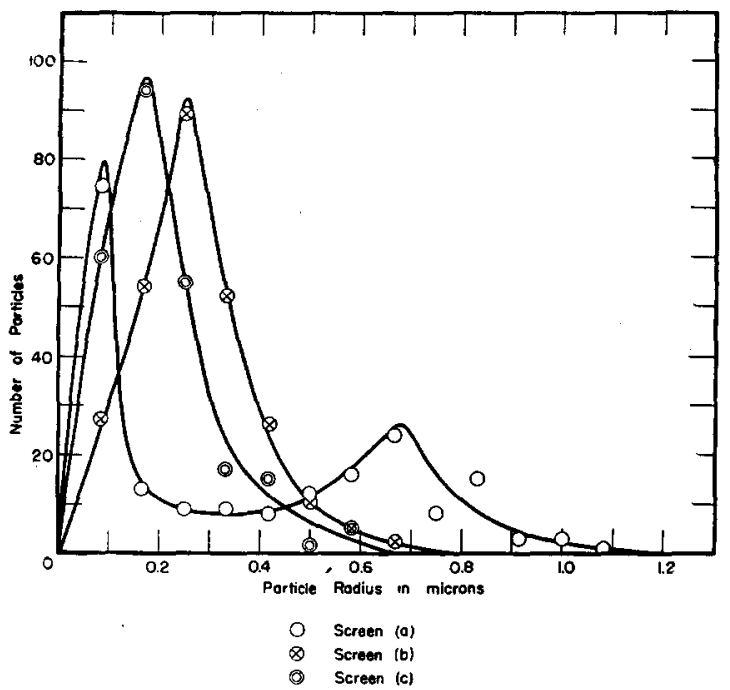

FIG. 3. Size-frequency distributions of particles in a rosin smoke charged by means of a cotton cloth at high potential.

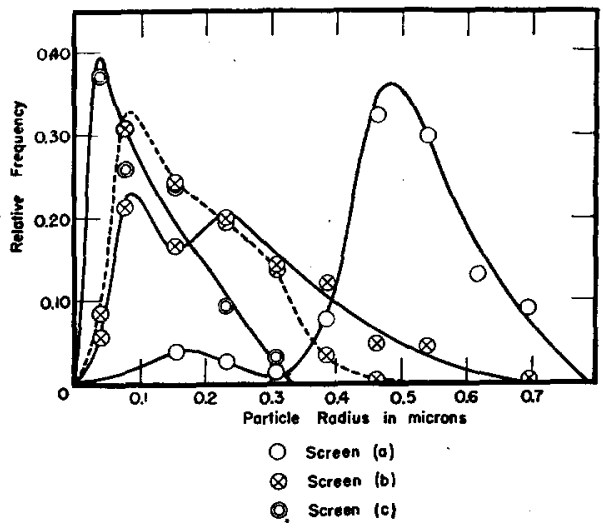

FIG. 4. Size-frequency distributions of particles in a rosin smoke charged by means of a plate coated with rayon flock fibers.

region. The lower plate was provided with a metallic collector $H$ which was mounted in the plane of this plate and insulated from it. This collector was made movable along the direction of wind flow in order to measure, by means of a galvanometer, the iron currents emitted by the charging device as a function of the position of the collector. The air-stream outlet consisted of two sections which ended finally in a large circular cross-section. A four-bladed fan, run from a variable speed motor, was placed just inside the outlet section $N$; this fan produced air velocities at the center of the precipitation 


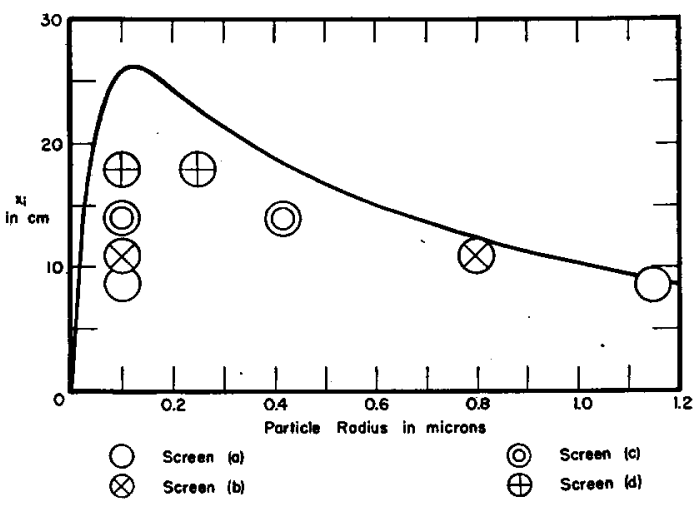

FIG. 5. Average particle radius as a function of point of precipitation; charging device was a heated filament.

chamber of 8 to $25 \mathrm{~cm} / \mathrm{sec}$. The velocities which were used in the runs described below were 12 to $15 \mathrm{~cm} / \mathrm{sec}$.

A uniform downward current of ions of about $10^{-8}$ ampere $/ \mathrm{cm}^{2}$ must be obtained in order to charge the smoke particles. Of the large number of devices which were tested for this purpose, the three most promising were investigated in detail

TABLE I. Summary of experimental results.

\begin{tabular}{|c|c|c|c|}
\hline \multicolumn{4}{|c|}{ Run 1; Charging device: heated filament } \\
\hline \multirow[b]{2}{*}{$\begin{array}{l}\text { Screen } \\
\text { (a) }\end{array}$} & Particles & & Position of maxima \\
\hline & $\begin{array}{c}\text { measured } \\
176\end{array}$ & $\begin{array}{c}x_{i} \text { in } \mathrm{cm} \\
8.7\end{array}$ & $\begin{array}{c}\text { in microns } \\
0.1 \\
1.15\end{array}$ \\
\hline \multirow[t]{2}{*}{ (b) } & 146 & 11.0 & $\quad 0.1$ \\
\hline & & & $\begin{array}{l}0.5,1.1 \\
\text { (Average, } 0.8 \text { ) }\end{array}$ \\
\hline (c) & 195 & 14.0 & $\begin{array}{l}0.1 \\
0.42\end{array}$ \\
\hline \multirow[t]{3}{*}{ (d) } & 99 & 18.0 & $\begin{array}{l}0.1 \\
0.25\end{array}$ \\
\hline & Run 2 ; Chat & device & otton cloth \\
\hline & & & $\begin{array}{l}\text { Position of } \\
\text { maxima and } \\
\text { half width at }\end{array}$ \\
\hline $\begin{array}{l}\text { Screen } \\
\text { (a) }\end{array}$ & $\begin{array}{c}\text { Particles } \\
\text { measured } \\
196\end{array}$ & $x_{i}$ in cm & $\begin{array}{l}\text { half maximum, } \\
\text { in microns } \\
0.08 \pm 0.05 \\
0.69 \pm 0.13\end{array}$ \\
\hline \multirow[t]{2}{*}{$\begin{array}{l}\text { (b) } \\
\text { (c) }\end{array}$} & $\begin{array}{l}265 \\
248\end{array}$ & $\begin{array}{l}12.0 \\
15.0\end{array}$ & $\begin{array}{l}0.25 \pm 0.08 \\
0.17 \pm 0.10\end{array}$ \\
\hline & \multicolumn{3}{|c|}{ Run 3; Charging device: rayon flock fibers } \\
\hline \multirow[b]{2}{*}{$\begin{array}{l}\text { Screen } \\
\text { (a) }\end{array}$} & Particles & & $\begin{array}{l}\text { Position of } \\
\text { maxima }\end{array}$ \\
\hline & $\begin{array}{c}\text { measured } \\
77\end{array}$ & $\begin{array}{c}x_{i} \text { in } \mathrm{cm} \\
4.0\end{array}$ & $\begin{array}{l}\text { in microns } \\
0.16 \\
0.48\end{array}$ \\
\hline \multirow[t]{2}{*}{ (b) } & 229 & 6.0 & $\begin{array}{l}0.08 \\
0.23\end{array}$ \\
\hline & 452 & 6.0 & $\begin{array}{l}0.09 \\
(0.2 \text { to } 0.3)\end{array}$ \\
\hline (c) & 97 & 8.6 & 0.05 \\
\hline
\end{tabular}

VOLUME 18, JANUARY, 1947

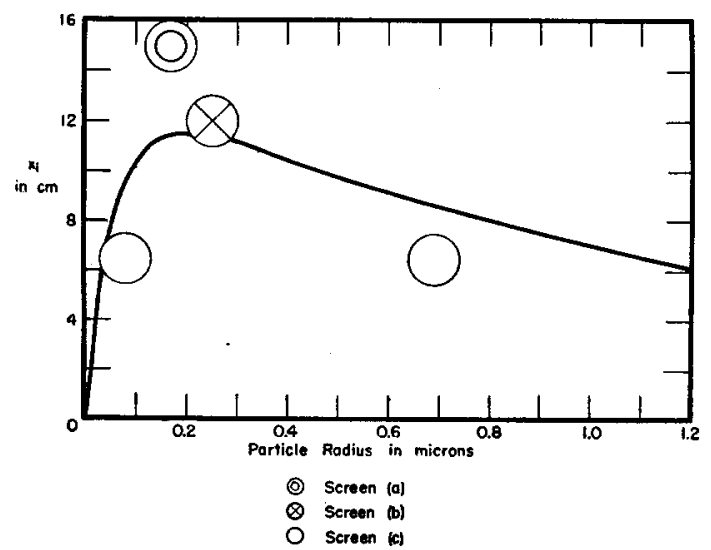

FIG. 6. Average particle radius as a function of point of precipitation; charging device was a cotton cloth.

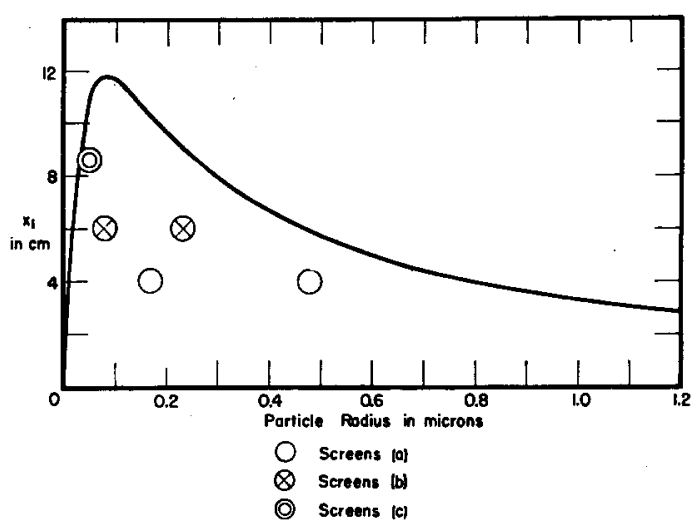

FIG. 7: Average particle radius as a function of point of precipitation; charging device was made from rayon flock fibers.

by studies of the size-frequency distribution of the smoke particles precipitated at various points in the apparatus. The first (Run 1) consisted of a small heated Nichrome coil which was supported by a short quartz rod and jacketed by a Pyrex compressed air cooler. Immediately below this coiled filament was placed a wire screen (15-50 meshes/in. ${ }^{2}$ ) which was allowed to remain at "floating" potential. The filament was heated by means of a filament transformer and was maintained at a potential of about $-5 \mathrm{kv}$. The second charging device (Run 2) consisted of a small area $(1 \mathrm{~cm} \times 2.5 \mathrm{~cm})$ of cotton bird'seye toweling which was placed in contact with a metal plate at -4 to $-10 \mathrm{kv}$. The third charging device (Run 3) consisted of a small aluminum plate maintained at $-14 \mathrm{kv}$ and covered sparsely with rayon flock fibers (about $250 / \mathrm{cm}^{2}$ ) attached 
normal to it. ${ }^{6}$ This third charging device gave the most nearly reproducible and uniform charging conditions of all of those which were tested.

For studies with the electron microscope, collodion-covered screens were placed on the lower plate of the precipitation apparatus at various distances from the charging region. These screens were $6-\mathrm{mm}$ circles cut from 350mesh brass screen, and were coated with a collodion film about 0.1 micron in thickness.

\section{EXPERIMENTAL PROCEDURE}

The wind velocity $v_{0}$ was measured in the following manner: a thin stream of smoke was allowed to flow down the center of the precipitation chamber. A disturbance was given to the stream by a slight movement of the inlet tube, and the time required for this disturbance to move a known distance was measured.

The ion current in the charging region was measured at various positions by means of the movable collector plate; it was found to be fairly uniform and limited to a small region. The extent of the charging region, extrapolated to the height of the smoke stream above the lower plate, divided by the velocity of the stream was taken as the time of charging, $t$. The average ion current per unit area in the charging region was taken as the ion current density, $i$.

The rosin smokes were prepared by heating about $70 \mathrm{mg}$ of rosin in a small copper pan; the rosin condensed into a smoke consisting of approximately spherical particles of radius $0.05<a$ $<3$ microns. The smoke was collected in the large flask and was forced into the apparatus by a slow stream of compressed air. When the charging and deflecting potentials were turned on, the smoke stream was observed to curve down slightly and fan out into a curtain of smoke dropping to the lower plate. The smoke thus deposited a narrow track of rosin on the lower plate where, for the purpose of electron microscopy, the small collodion-covered collectors were placed.

\footnotetext{
${ }^{6}$ Flock is a preparation of textile fibers cut to uniform, short lengths. In order to prepare this charging device the required density of flock was placed on a grounded conducting plate. The aluminum plate, wet with Glyptal, was suspended above the grounded plate and charged to about $-15 \mathrm{kv}$ in order to deposit the fibers in an erect position orr the charged plate. The potential was left on until the Glyptal hardened.
}

Photographs were taken of the smoke deposits on the collodion-covered screens with the use of the electron microscope designed at the California Institute of Technology by Professor W. V. Houston and Dr. J. H. Bradner, and the RCA electron microscope at the Department of Physics of the University of California at Berkeley. Enlargements of the electron microscope photographs were made to a total linear magnification of about 6000 . For spherical particles the diameter was measured ; for elliptical and slightly irregular particles the average of the largest and smallest diameters was taken. Nearly all of the rosin smoke particles were approximately spherical except for the larger particles obtained in Run 1, in which the heated filament was used as a charging device. These highly irregular particles were divided into spherical or approximately elliptical sections and measured as independent parts. Although this idealization is clearly not a good approximation, a convenient method of comparing particle sizes was obtained by this method. There is no satisfactory treatment of the law of fall for particles of irregular shape. From the measurements of particle size, the size-frequency distribution curves were prepared by plotting the number of particles of a given size as ordinate against the radius of the particle in microns as abscissa, and then drawing a smooth curve through the points.

\section{RESULTS}

The size-frequency distribution curves obtained in the three runs are shown in Figs. 2a, $2 b, 3$, and 4 . The experimental results are summarized in Table I. On Screen (b) of Run 1 two maxima appeared for $a>0.3$ microns whereas the theory required only the one shown by most of the other experimental results. The average of these two peaks was taken. It is to be noted that the numbers of particles of given size determining parts of this and some of the other distribution curves are too small to yield satisfactory statistical distributions. As a result the method of interpolation by which some parts of the curves should be obtained is not unambiguous. The photographed area of Screen (b) of Run 3 was divided into two parts, and the particles on these two parts were counted separately in order to determine whether the result- 
ing size-frequency distribution curves were in satisfactory agreement. The results, shown in Fig. 4 and Table $I$ are in good agreement, but it is quite clear that a large number of particles must be counted before satisfactory distributions are obtained.

The experimental results are compared with the theoretical curves, calculated as described in the Appendix, in Figs. 5, 6, and 7. The numerical expressions from which the curves were calculated are, for Run 1,

$$
x_{i}=\frac{1660 a_{\mu}}{\left(1+0.082 / a_{\mu}\right) Q} \text { where } Q=123 a_{\mu}{ }^{2}+23 a_{\mu} ;
$$

for Run 2,

$$
x_{i}=\frac{1840 a_{\mu}}{\left(1+0.082 / a_{\mu}\right) Q} \text { where } Q=157 a_{\mu}{ }^{2}+82 a_{\mu} ;
$$

and, for Run 3,

$$
x_{i}=\frac{1840 a_{\mu}}{\left(1+0.082 / a_{\mu}\right) Q} \text { where } Q=476 a_{\mu}^{2}+39 a_{\mu},
$$

where $a_{\mu}$ is the particle radius in microns. The agreement between the curves and the experimental points is not too good. However, the general character of the theoretical curves is reproduced by the experiments, for example, the precipitation of both large and small particles at a given point as required by the theory appears to be verified.

In order to test whether the original smoke sample itself contained an abnormally large number of small particles, the whole thread of smoke was swept repeatedly across a collodion-covered screen by variation of the corona potential, until a satisfactory density of particles was obtained. Electron microscope photographs of this sample did not show a predominant number of particles of radius less than 0.2 micron; the size-frequency distribution curve for the unseparated smoke showed a single maximum at about 1 micron.

Considerable experimental difficulties were encountered in the use of the various charging devices. The filament heated the charging region considerably, and even though approximate thermal equilibrium had been reached before the run was started some slight thermal unsteadiness in the precipitation conditions persisted. The cotton cloth charging device produced results which were quite unreproducible; however, no variations in the charging conditions were usually observed during a single run. The conducting plate covered with the rayon flock fibers was probably the most satisfactory charging device which was tested. These fibers undoubtedly retained their relative orientation for successive runs better than did the cloth fibers. The appearance of the track of rosin precipitated on the lower plate was very similar for successive runs with the flock fiber charging device, although a series of five successive runs under as nearly the same conditions as possible gave results which agreed rather poorly.

The reasons for this poor agreement probably include (a) variations in the initial charges on the particles, (b) turbulence in the wind flow in the tunnel, (c) irregularities in the particle shape, (d) fluctuations in the charging current, and (e) statistical variations in the charging. The effects of variation of the initial charges of the particles were estimated by measurement of the maximum deflection of the smoke stream in a uniform field in the absence of the precipitating current. The maximum deflection observed corresponded to a maximum initial charge of $\pm 8 e$ on a particle of radius 0.5 micron, or $\pm 34 e$ on a particle of radius 2 microns. Turbulence is extremely difficult to eliminate completely, and its effect was apparent on careful visual observation under the best conditions which we obtained; it may have been caused, in part, by electrical wind effects. Fluctuations in the charging current of 1 to 2 percent were detected with a probe which was connected to an electrometer circuit. The effect of irregularities in the shapes of the particles and of statistical variations in charging could not be estimated.

\section{ACKNOWLEDGMENT}

Our thanks are due Professor Linus Pauling, who suggested this study, for his interest, advice, and encouragement. We are also indebted to Mr. George Standart for assistance with part of the experimental work, and to Dr. R. V. Langmuir and Mr. R. Rau of the California Institute of Technology, Mr. A. E. Smith of the Shell Development Company, and Mr. A. W. Einarsson of the University of California at Berkeley 
for their assistance in helping us to obtain electron microscope photographs.

\section{APPENDIX: THEORETICAL SECTION}

A particle which has a radius $a$ and charge $Q e$ and is swept along a horizontal rectangular channel (see Fig. 8) between two charged plates has a downward velocity $v^{\prime}$ because of the gravitational and electrostatic forces; $m g+E_{0} Q e$;

$$
v^{\prime}=\frac{m g+E_{0} Q e}{6 \pi n a}\left(1+\frac{A \lambda}{a}\right)
$$

where $\eta$ is the viscosity of the gas; $A$ is the Cunningham constant ${ }^{7}$; and $\lambda$ is the mean free path of the gas molecules.

The problem of the gas velocity at any point in a tube of rectangular cross section has been solved by Cornish. ${ }^{8}$ In a tube with cross-sectional width $c$ and height $d$ in which the velocity on the axis $(y=z=0)$ is $v_{0}$, the velocity of gas flow along any line parallel to the axis is

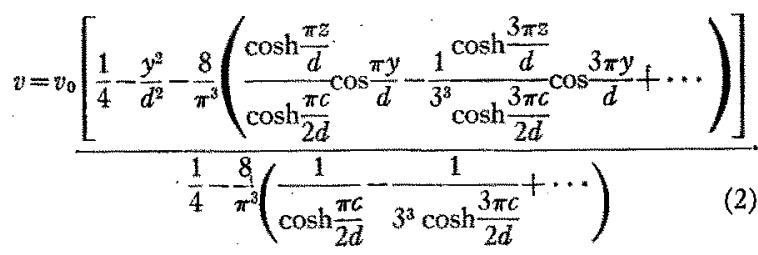

The horizontal distance, $x_{i}$, from the point at which the particles are charged to the point at which they strike the lower plate may then be shown, by integration of the equation $d x=\left(v / v^{\prime}\right) d y$, to be

$$
\begin{aligned}
x_{i}=\frac{6 \pi \eta a v_{0}}{\left(m g+E_{0} Q e\right)}\left(1+\frac{A \lambda}{a}\right)^{\frac{2}{d}}\left(1-\frac{2 l}{3 d}\right) \\
\times\left\{1+\frac{32}{\pi^{3} \cosh \frac{\pi c}{2 d}\left[1+\frac{32}{\pi^{3} \cosh \frac{\pi c}{2 d}}\right]}\right. \\
\left.\quad \times\left[1-\frac{d^{2}\left(1-\cos \frac{\pi l}{d}\right) \cosh \frac{\pi z}{d}}{2 \pi l^{2}\left(1-\frac{2 l}{3 d}\right)}\right]+\cdots\right\},
\end{aligned}
$$

where $l$ is the height above the lower plate at which the stream of particles is charged. For the conditions under which the experiments were carried out $E_{0} Q e \gg m g$ and $z=0$, and an adequate approximation to Eq. (3) is

$$
x_{i}=\frac{6 \pi \eta a v_{0}}{E_{0} Q e\left(1+\frac{A \lambda}{a}\right)^{d}}\left(1-\frac{2 l}{3 d}\right) .
$$

This equation would be suitable for calculation if we knew the number of charges, $Q$, as a function of the particle radius and known or measurable quantities.

A theory of the charging of a spherical particle of radius $a$ and dielectric constant $K$ passing through a downward current of ions has been derived by Rohmann ${ }^{5}$ under the assumptions that (1) difusion of charges to the particle is

${ }^{7}$ E. Cunningham, Proc. Roy. Soc. A83, 357 (1910).

8 R. J. Cornish, Proc. Roy. Soc. A120, 691 (1928).

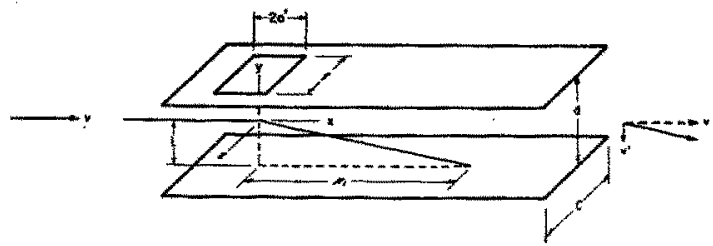

Fra. 8.

negligible, (2) electrical image forces between an ion approaching a particle and the particle are negligible, and (3) all of the incident ions give up their charge to the particle. His expression for the charge $Q_{f} e$ acquired in time $t$ by an initially uncharged particle may be written in the form

$$
Q_{f}=\left(1+\frac{2(K-1)}{K+2}\right) E_{1} a^{2} \frac{\pi i t}{E_{1}+\pi i t}
$$

where $i$ is the ion current per unit area and $E_{1}$ is the field intensity in the charged region.

No satisfactory theory has been developed for the initial stage of the charging of a spherical particle of low dielectric constant by diffusion. The final stage of diffusion charging was investigated theoretically and experimentally by Arendt and Kallmann. ${ }^{9}$. Their work, and also the experimental work of Deutsch ${ }^{10}$ and Schweitzer ${ }^{12}$ showed that the saturation charge taken up by oil drops of radius from 0.1 micron to 10 microns in a space charge of the order of $10^{5}$ charges $/ \mathrm{cm}^{3}$ is proportional to the particle radius. Ladenburg ${ }^{13}$ pointed out that in the process of precipitation of smoke particles in a charging field there are two distinct charging mechanisms, one, important for the larger particles, giving a charge proportional to $a^{2}$ as described in the preceding paragraph, and another, important for the smaller particles, giving a charge proportional to $a$. This second mechanism is called diffusion charging although it includes, in addition to ordinary diffusion of ions to the particle, the effect of image forces on the charge approaching the dielectric surface of the particle and the effect of the net charge on the diffusion of ions to the particle. We have assumed that the number of charges diffusing to a particle of radius $a$ in a time $t$ and in an ion atmosphere of volume density $f$ is

$$
Q_{d}=C f t a .
$$

The value of the proportionality constant was determined to be $C=0.07$ by extrapolation to zero time of the experimental curves for the charging of oil drops as a function of time, as given by Arendt and Kallmann. ${ }^{9}$ Unfortunately, Arendt and Kallmann did not obtain data for times less than about $4 \mathrm{~min}$., and consequently the constant $C$ cannot a priori be regarded as determined even to the correct order of magnitude for the time intervals of interest $(i \sim 0.2 \mathrm{sec}$.$) . We shall, nevertheless, use the resulting$ expression for calculation of $Q_{d}$ because our results described above indicate that the resulting term in the

${ }^{9} \mathrm{P}$. Arendt and H. Kallmann, Zeits. f. Physik 35, 421 (1926).

is W. Deutsch, Zeits, f. tech. Physik 7, 623 (1926).

${ }^{11}$ H. Schweitzer, Ann. d. Physik [5] 4, 33 (1930).

${ }^{12}$ R. Ladenburg, Ann. der Physik [5] 4, 863 (1930). 
charging law is approximately correct, and is almost certainly of the correct order of magnitude. Since the ion density is given by the relation $f=i /\left(E_{1} u e\right)$, where $u$ is the mobility of the ions, Eq. (6) therefore becomes

$$
Q_{d e}=0.07 \mathrm{iat} / E_{1} u \text {. }
$$

In the absence of a complete mathematical treatment of the charging process, we shall write the expression for the total charge at a time $t$ on a spherical particle of radius $a$ in the form

$$
Q e=Q_{f} e+Q_{d} e,
$$

where $Q_{f} e$ and $Q_{d} e$ are given by Eqs. (5) and (7), respectively. In this and the preceding equations the effect of the comparatively small initial charge on the particle has been omitted. Although the two charging processes are probably not independent, and the expressions for the two processes therefore not strictly additive, it may be pointed out that because of the different dependence of the two processes on the particle radius, Eq. (7) represents the important term for the smaller particles, whereas Eq. (6) represents the important term for the larger particles.
The value of $Q e$ from Eq. (8) may be substituted into Eq. (4) in order to calculate the distance along the direction of gas flow from the center of the charging region to the point of precipitation of a particle of radius $a$. In this calculation we have made the additional assumption that the charging, which occurs over the distance $v_{0} t=2 a^{\prime}$ (Fig. 8), may be approximated by complete charging at a point at the center of the charging region.

In some of the experiments the field in the charging region was considerably higher than that between the deflecting plates of the rectangular precipitation chamber. The average field in the charging region in this case was calculated from an approximate solution of the twodimensional potential distribution by expansion in rectangular harmonics. For the case (Run 3, above) in which this treatment was applied, the field thus calculated was about 7 percent smaller than that obtained from the relation $E_{1}=-V_{1} / d$, where $V_{1}$ is the potential of the upper plate of the rectangular tube. The effect of space charge in decreasing the field intensity in the charging region was small enough to be neglected.

\title{
Dislocation Theory as Applied by N.A.C.A. to the Creep of Metals
}

\author{
A. S. Nowick and E. S. Machlin \\ Aircraft Engine Research Laboratory, Cleveland, Ohio
}

(Received September 9, 1946)

\begin{abstract}
An equation for the steady-state rate of creep of pure annealed polycrystalline metals is derived through dislocation theory and the theory of rate processes. The rate of generation of dislocations is shown to be the rate-determining process. A specific mechanism for the generation of a dislocation is presented, from which the heat and entropy of activation of the process are expressed in terms of physical constants of the material. In addition the lowering under stress of the potential energy barrier, which yields the stress-dependent term, is given in terms of constants of the material and a "back-stress" term. The creep equation thus obtained is found to be in good agreement with data in the literature.
\end{abstract}

\section{INTRODUCTION}

$\mathrm{C}$ REEP of metals is generally subdivided into three stages, as shown in Fig. 1. The initial stage, in which the slope of the elongationversus-time curve (the rate of creep) is rapidly decreasing, is commonly designated the primary or transient stage; secondary or steady-state creep refers to the straight-line portion of the creep curve. After a sufficient length of time, the rate of creep increases in the region designated the tertiary stage.

The criterion generally used to evaluate the creep property of a given material at a particular stress and temperature is the steady-state creep rate. One of the first attempts to analyze steady- state creep by other than empirical methods was by Kanter. ${ }^{1}$ Later, Kauzmann ${ }^{2}$ applied Eyring's theory of liquid flow, which is a special application of the theory of rate processes, to the steady-state flow of metals. The problem of creep has also been attacked by the use of the theory of dislocations. A survey of results obtained thus far by this approach is reported by Seitz and Read. ${ }^{3}$ Although these three treatments predict equations for the steady-state creep rate that show the correct dependence on stress and temperature, quantitative predictions cannot be

1 J. J. Kanter, Trans. A.I.M.E. 131, 385 (1938).

2 W. Kauzmann, Trans. A.I.M.E. 143, 57 (1941).

${ }^{3}$ F. Seitz and T. A. Read, J. App. Phys. 12, 100, 170, 470,538 (1941). 